[SPECIAL EDITIONS on "SMART TEXTILES"-Technical Paper】

\title{
Smart Clothing Integrates Both Health and Maximum Comfort
}

\author{
Wen-Hsien Sun* and Jing-Wen Tang \\ Material and Chemical Research Laboratories, Industrial technology Research Institute, Taiwan
}

\begin{abstract}
ITRI's unique clothing, iSmartwear, incorporates washable technology and breaks the mold of traditionally tight, uncomfortable, clothing that not only has poor heat dissipation, weak endurance, but also lower sensor sensitivity. iSmartweaR is ITRI's truly practical solution to address the problem of correct detection of vital sign signals from the body of a smart clothing wearer. It incurs virtually no negative issue in comfort and convenience at all.

(Received 12 July, 2017; Accepted 20 November, 2017)
\end{abstract}

\section{Introduction}

Smart clothing has been around for a while. They come in to provide a means for the collection of the wearer's basic vital signs of pulse and respiration rates. Such data can be used as the basis for analysis to provide useful reference information like calorie burnt to the wearer regarding his or her general biological conditions during, mostly for example, exercise sessions. Such are helpful in the sense of general healthcare for smart clothing consumer. However, such simple tasks as collecting signals from the human body for the determination of the basic heart and respiration rates have proved to be tricky if true practicality is desired. Other than iSmartweaR, all other technologies known to ITRI rely on the use of electrodes that must be in direct and firm contact with skin so that electric cardiac signals can be detected by means of electronic circuitry. Using the body electrical signal, pulse rate can be determined with relative ease - but not respiration rate.

Such technologies are impractical as they are uncomfortable to wear because of the electrodes[1-3]. Also due to electrodes, it's difficult to collect data reliably for prolonged time. Further, it's difficult to derive respiration rate from the signal picked up by skin electrodes. A separate means - of different technological nature - must be used to deal with respiration detection.In strict technical sense, iSmartweaR is a smart clothing technology to detect wearer pulse and respiration rates using radio frequency Doppler radar[4,5]. It is non-contact, relying on the detection of minute body movements reflected on the skin surface that are caused by the beating of artery and the contraction and expansion of the chest caused by breathing[6,7].

\section{Development of New Type of Smart Clothing}

In a broader sense, iSmartweaR is able to provide correct detection of vital signals for prolonged time periods, with the basic data subject to secondary analyses for the derivation of useful information for the wearer's reference on a variety of health-related information.Distinctly and uniquely non-contact in nature, iSmartweaR provides a truly practical basis for implementing one useful feature, the detection of body vital signs for smart clothing. With the availability of reliable and continuous feed of wearer basis vital sign information, iSmartweaR opens up a series of application possibilities. These start with the basic applications in personal general healthcare assistance functionalities and beyond:

- Personalized biological vital signs measurement and monitoring: Pulse and respiration rates and gross activity sensing and measurement.

- Personal health maintenance and improvement Biological conditions monitoring during exercise

\# corresponding author: Sun Wen-Hsien (E-mail: sws0928@itri.org.tw) 
sessions; driving fatigue alert, sleep quality analysis and improvement and emotion management.

- Biological conditions monitoring for medical care: Monitor of patient's biological conditions and analysis of sleep disorder.

- Professional rescue and caring in specific emergency incidents: Biological conditions monitoring and analysis of special police and firefighter trainees during training sessions and rescue workers' own health monitoring and irregularity alerts.

From the perspective of specific applications group, and considering the benefit of this iSmartweaR technology to be free from the inconvenient need for direct contact with user skin, as well as hazard-free low-power microwave (uW category compared to regular mobile phones' $250-500 \mathrm{~mW}$ ) that meets medical instrument requirements, iSmartweaR is particularly suitable for the following applications:

- Biological vital signs measurement and preventive care of sudden death of infants.

- Professional driver health status analysis and fatigue sensing and warning.

- Biological conditions monitoring for athletes during training sessions

- Health conditions monitoring and chronic diseases caring for elderly

- Health conditions monitoring and anomaly warning for professionals (police, firefighter and rescue worker)

As a whole, iSmartweaR constitutes the backbone of a whole spectrum of healthcare assistance applications. They include general health monitoring applications requiring no FDA regulations such as playing the role of an exercise companion to provide basic guidance for smooth and effective exercise sessions, both for regular people and professional athletes. However, iSmartweaR can also be involved in vigorous applications such as patient vital sign basic monitoring, which are regulated by the authorities. iSmartweaR's clever use of microwave radar makes all these applications practically possible.

Key to iSmartweaR smart clothing applications' practical use in the detection of wearer basic vital signs of pulse and respiratory rates is the microwave Doppler radar.

\subsection{NPNS Doppler Radar}

ITRI has developed an industry-leading
Nanosecond Pulse Near-field Sensing (NPNS) technology that operates a low-power radar. Under NPNS, the radar operates Doppler-effect target detection, discerning minute skin surface movements caused by heart beats and respirations from radar waves reflected from the skin.

Operating at microwave frequency, the NPNS Doppler radar can be positioned so close to the skin yet is capable to correctly pick up the radar reflection. Further, running a tiny power rating to cause no concern of negative health impact and also save energy for extended time of use on battery, the microwave is able to penetrate through fabrics of clothing, like an underwear, worn above the skin and below the smart clothing that hosts the radar antenna.

As long as a continuous stream of radar reflection signals can be sensed by the antenna, an algorithm can then be responsible for screening out the pulse rate and the relatively much lower rate of respiration, both from the signals picked up by the antenna.

ITRI's NPNS Doppler radar is key to iSmartweaR's practical non - contact sensing functionality. It is easily integrated with contemporary consumer digital system such that iSmartweaR smart clothing can be conveniently useful for many applications. In the future it can be integrated with additional modules such as for detecting blood pressure and cardiac parameters to provide more sophisticated usefulness. Smartphone, tablet computers and other handheld smart devices can provide user interface for the technology, communicating wirelessly through, Bluetooth for example, with the compact mobile electronics of an iSmartweaR smart clothing system.

\subsection{Material for Antenna Durable for Washing}

Critical to an iSmartweaR Doppler radar is the technique to make an antenna that is compatible with normal fabrics used for the clothing. The antenna is made by painting an electrically conductive paste shaped to the desired antenna pattern on the fabric. The conductive paste contains a nanometer-sized powder that is electrically conductive. The painted antenna is then cured and set.

iSmartweaR's antenna is highly electrically conductive to meet the need for use as the microwave radar. As it is painted into the fibers of the fabric, it is also flexible, and extremely durable for washing. This is important because many of an iSmartweaR smart clothing's applications involve high-caliber exercises. The wearer sweets, and the clothing must be cleaned 
by washing.

Compared to conventional technologies that implement vapor deposition or sputtering of conductor material onto the fabric, or chemically bonding silver molecules onto fibers of the fabric, iSmartweaR is much simple and therefore costs lower, an important factor for commercialization. FIG. 1 shows NPNS Doppler radar module (top) and durable antenna pad (center) for an iSmartwear smart clothing (bottom).

In a typical implementation of a smart clothing using iSmartweaR, radar antenna is integrated on the clothing, for example, a sports jersey. Normally it is located at the upper chest at left, as is shown in the blue patch of FIg 2 .

The antenna is electrically linked to a body electronics that the wearer straps on the waist, as seen on the right side. The controller is electrically connected to the antenna via a pair of button-type connectors, which has a pair of conductor wires woven into the fabrics of the smart jersey.

The antenna radiates radar waves to the skin of the wearer just under the blue patch that has antenna embedded. Without requiring any physical contact to the skin, the radar antenna receives radio frequency microwave signal reflected back from the skin and sends back to the body electronics for analysis. The algorithm discriminates skin motion signals and
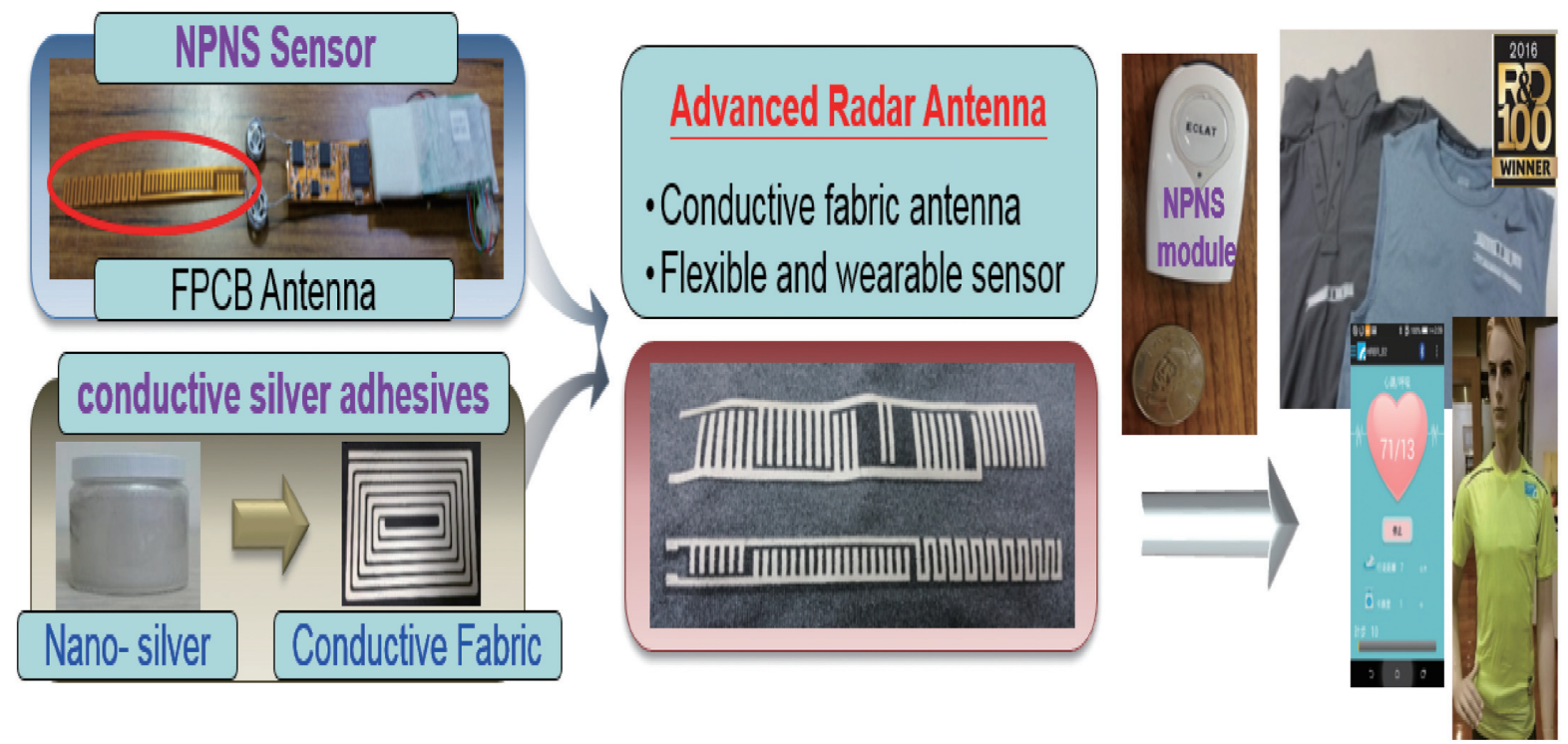

Fig. 1 Advanced radar antenna.
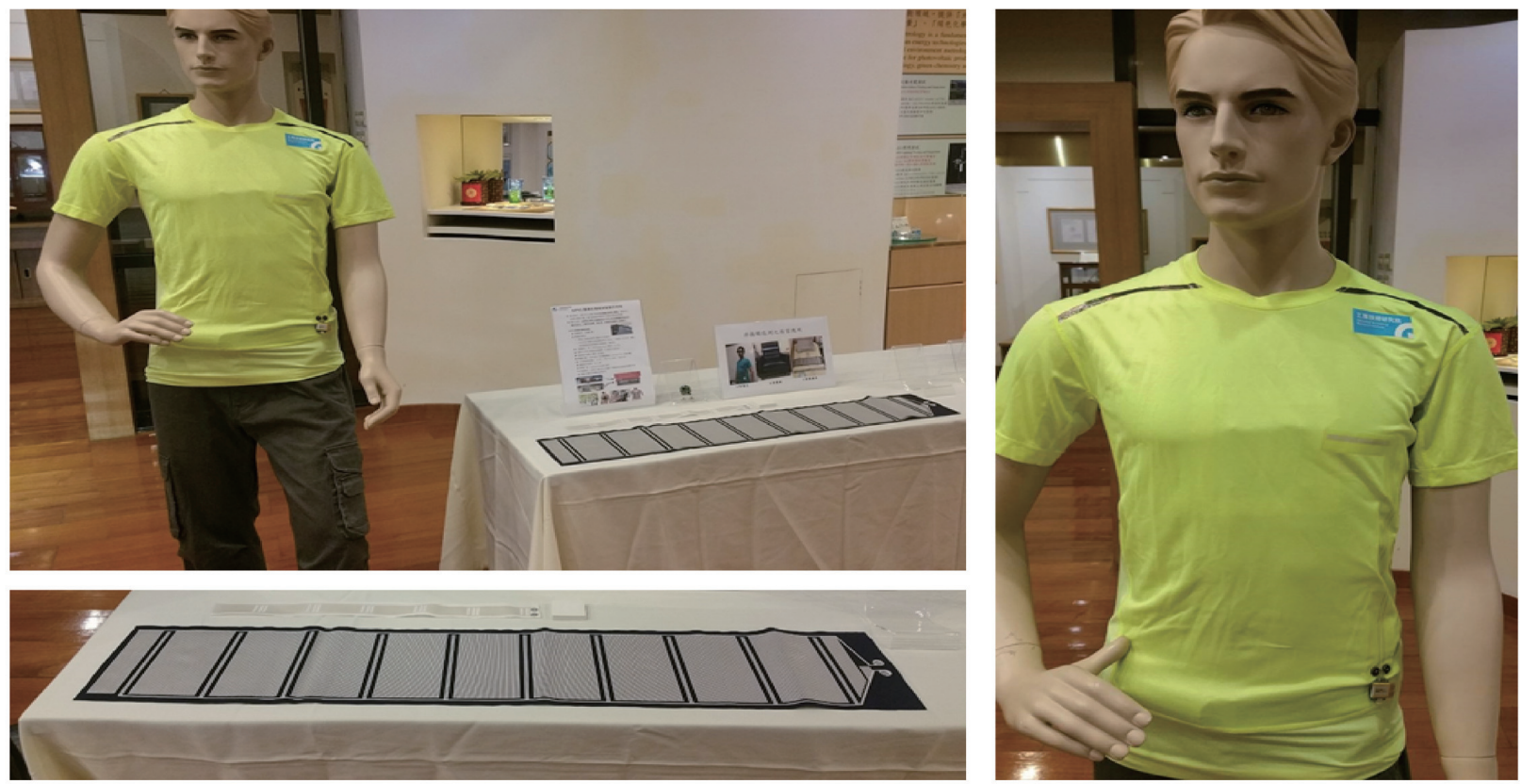

Fig. 2 ITRI's iSmartwear, designed for both comfort and health. 
screens out the heart beat and respiration signals.

The technology does not rely on constant firm contact of a pair of electrodes to the skin, thereby providing best possible wearer comfort and sustaining reliable data collection. ITRI has demonstrated an iSmartweaR smart clothing that is characterized by the following features:

- Type of smart wear: Fabric cloth

- Electrically conductive fabrics: Post processing of fabric for screen printing/transferred printing to make a flexible antenna patch, surface resistance $<10 \Omega / \square$, signal-to-noise ratio $>20 \mathrm{~dB}$ after 50 washing cycles, certified for bio-compatibility and achieves knit ware functionality compliance.

- Heartbeat detection: 48-240 beats/min (precision $95 \%$ )

- Pacing calculation and calorie consumption estimation: 3-axis G-sensor

- Wireless data linkage: Bluetooth 4.0 BLE

- Power supply: $250 \mathrm{mAh}$ rechargeable Li-ion battery (POGO Pin charging interface)

- Operating environment: $5^{\circ} \mathrm{XC}-70{ }^{\circ} \mathrm{XC} / 20 \%$ $80 \%$ relative humidity

- Continuous operation: 12 hours

- Software interface: App of Smartphone/tablet

- User display: Realtime heart rate/paces/ distance/calorie consumption and trend for exercise session
- Clothing-antenna-controller full integration

\section{Property of iSmartweaR}

iSmartweaR is the only smart clothing technology to host a truly non-contact vital sign detection technique. Table 1 is comparing iSmartweaR to other smart wear technologies. It is simple, straightforward, yet cheap, effective and, most of all, really practical for the average user. Such practicality is the only reason to really make such healthcare assistance technologies really useful.

All conventional smart clothing technologies known to ITRI project team are wired - they require at least a pair of two electrodes to electrically wiring the sensor circuitry to the wearer's body. The sensor circuitry only works with a complete circuit, and this needs the two sensor electrodes to contact the skin properly. Without good contact, no data can be collected, and this means no vital sign information. Thus, firm and constant electrode-skin contact is critical to the functionality of the smart clothing.

This compares to ITRI iSmartwerR's wireless for the collection of wearer vital signs. iSmartweaR is "wireless" because it uses radar signal to detect wearer's vital signal. No sensor electrode means user comfort for long sessions of use. The radar power is so low that it does not constitute any issue to wearer health. It's so low that it drains only little power from

Table 1 Comparing iSmartweaR to other smart wear technologies

\begin{tabular}{|c|c|c|c|c|c|}
\hline & Non-contact & \multicolumn{4}{|c|}{ Skin Electrodes } \\
\hline Developer & $\begin{array}{c}\text { ITRI } \\
\text { (iSmartweaR) }\end{array}$ & Infeneon & $\begin{array}{c}\text { VivoMetrics, } \\
\text { USA }\end{array}$ & Philips & Sensatex \\
\hline Heart Rate & Yes & Yes & Yes & Yes & Yes \\
\hline $\begin{array}{l}\text { Respiration } \\
\text { Rate }\end{array}$ & Yes & No & No & Yes & Yes \\
\hline $\begin{array}{l}\text { Electrodes } \\
\text { on Skin }\end{array}$ & None & Yes & Yes & Yes & Yes \\
\hline $\begin{array}{l}\text { Type of } \\
\text { Electrode }\end{array}$ & Not applicable & $\begin{array}{c}\text { Conductive } \\
\text { rubber }\end{array}$ & $\begin{array}{c}\text { Conductive } \\
\text { fabrics }\end{array}$ & Smart textile & Metallic \\
\hline $\begin{array}{l}\text { Available } \\
\text { System } \\
\text { Integration }\end{array}$ & $\begin{array}{c}\text { Portable device } \\
\text { (Smart } \\
\text { Phone/TabletPC) }\end{array}$ & 3C music player & $\begin{array}{l}\text { VivoMetrics } \\
\text { (Proprietary } \\
\text { closed system) }\end{array}$ & Smart phone & $\begin{array}{c}\text { Smart phone, } \\
\text { personal } \\
\text { computer }\end{array}$ \\
\hline
\end{tabular}


the battery, allowing practically prolonged time of service per battery charge. It's low yet still has a capability to penetrate fabrics that a wearer can even have underwear below the smart clothing. Most important of all radar detection of vital signs is so reliable regardless of wearer activity, like in sports with high physical activities.

iSmartweaR eliminates all discomfort and inconvenience from the useful application of personal vital sign signal collection. In all, iSmartweaR improves upon competitive smart clothing technologies by being truly practical to use. For an iSmartweaR smart clothing to deliver its best usefulness, it needs to discern wearer skin movement, surface activities reflecting pulses and heartbeats. It is therefore considered a limitation that the radar antenna be positioned at a location, for example, right next to an artery, preferably close to the surface of skin. However, such limitation is minor. With an iSmartweaR, the antenna comes mostly in the form of a patch that can be attached to the clothing via normal clothing fabrication means. For example, it can just be sewed to the clothing facing the upper chest close to the collarbone.

Useful in assisting healthcare, smart clothing has made an inroad. It senses basic body vital sign information for deriving biological information like calorie consumption that is more meaningful reference to the wearer. Though, heart and respiration information in smart clothing requires only the simple "rate," the number of heartbeats and respirations per minute. This is unlike in medical applications that need, for example, ECG waveforms requiring firm contact of more than two sensor electrodes with skin. To obtain these basic rate numbers, ITRI's RF radar is a much better implementation than conventional use of body electrodes because maintaining firm skin-electrode contact becomes an annoyance once prolonged in time.

\section{Conclusion}

Because of it's non-contact and fabric-penetration characteristics, ITRI's iSmartweaR provides a truly practical solution for smart clothing. Comfort yet reliable sensor outfit is now true. More, a spectrum of versatile healthcare assisting applications are now possible: wearer body motion monitoring useful in improved caring of both in- and out-patients of various illness.

\section{References}

1. J. C. Lin. (1975). Proc. IEEE, 63, 10, 1530.

2. J. C. Lin.(1992), 13, 6, 557-565.

3. C. Li, V. M. Lubecke, O. Boric-Lubecke, J. Lin. (2013), IEEE Trans. Microw. Theory Techn., 61, 5, 2046-2060.

4. Kong, H. and Jang, J. (2008). Langmuir, 24 (5), 2051 -2056 .

5. Tang, B., Wang, J., Xu, S., Afrin, T., Xu, W., Sun, L., \& Wang, X. (2011). J Colloid Interface Sci., 356 (2), $513-518$.

6. Li, Y., Hou, Y. Y., and Zou, Y. L. (2012). Fibers and Polymers, 13, 185-190.

7. Montazer, M., Alimohammadi, F., Shamei, A., \& Karim Rahimi, M. (2012). Carbohydrate Polymers,87, 1706-1712. 